Proceedings of the 2002 IEEE/RSJ

Intl. Conference on Intelligent Robots and Systems

EPFL, Lausanne, Switzerland • October 2002

\title{
Kinematic Inversion
}

\author{
A. Ramdane-Cherif ${ }^{1}$, B. Daachi ${ }^{2}$, A. Benallegue ${ }^{2}$ and N. Lévy ${ }^{1}$ \\ ${ }^{1}$ PRiSM, université de Versailles, France \\ ${ }^{2}$ Laboratoire de Robotique de Versailles, France \\ \{rca@prism.uvsq.fr, daachi@robot.uvsq.fr\}
}

\begin{abstract}
We propose a new contribution to solve the inverse kinematic problem of redundant robots subject to a set of criterion and constraints. Traditional methods are usually very complicated to use in real environment and present many problems such as high convergence time and possible instability. First, a study of the existent methods leads us to develop an on-line algorithm based on an adaptive neural network. This solution needs only a few iterations to converge, offers substantially better accuracy, verifies the repeatability propriety for the closed trajectory and avoids the computation of the inverse or pseudoinverse Jacobian matrix. Our approach guarantees a good minimization of any performance criterion subject to either equality or inequality constraints while achieving the end-effector task. Then, our method can solve the inverse kinematic problem of redundant robot for it to follow a desired trajectory while avoiding moved or fixed obstacles.
\end{abstract}

\section{Introduction}

In order to control a robot manipulator to accomplish a task, trajectory planning is required in advance or in real time. The desired trajectory is usually described in Cartesian coordinates and needs to converted to joint space for the purpose of analysing and controlling the system behavior.

The inverse kinematic model can generally be approached either by a geometrical or numerical solution. The geometric method [1][2] derives a closedform solution from the direct kinematic equations. For manipulators that do not have a closed-form solution like kinematically redundant manipulators, standard numerical procedures [3][4] solve the nonlinear differential equation at a discrete set of points.

Singularity and redundancy are two main difficulties in robot motion synthesis. At certain locations in joint space, the jacobian matrix loses rank. These points correspond to singular arm configurations. As the arm approaches these points in joint space, $J(\theta)$ becomes ill conditioned and the numerical inverse solution leads to very large joint velocities.

Kinematically redundant manipulators have recently been the subject of active research since their extra degrees of freedom allow more sophisticated motion than conventional manipulators. One of the problems in controlling redundant manipulators is a considerably increased computational complexity compared with non redundant robots. For a given workspace position, one solution has to be chosen among an infinite number of inverse kinematic solutions corresponding to all possible configurations. Some of previous works then use the redundant degrees of freedom to achieve some performance during the motion such as: avoiding obstacles, keeping the joint coordinates within their joint limits, avoiding singularities, improving dexterity, optimizing joint torques, minimizing manipulability and so on $[5][6][7][8]$.

Our approach is based upon an optimization scheme using a neural network. The neural network is adapted in the direction of decreasing a Lyapunov function to move the end effector to the desired position. This approach exploits the redundancy to achieve some objective functions, and to satisfy some inequality constraints while tracking the desired end-effector trajectory. Penality functions convert the constrained optimization problem into an unconstrained problem. This algorithm does not re quire to compute the inverse or pseudoinverse Jacobian matrix. This method provides an accurate solution with only a few iterations per input point even in singularities: the desired end-effector trajectory is good tracked, the performance function is well minimized and the additional inequality constraints are satisfied. The repeatability propriety is virefied which means that for the closed or cyclic trajectory the initial and final position and velocity are the same.

The organization of this paper is as follows. In section 2, we recall the kinematic formulation of robot manipulators. Section 3 is devoted to give some in- 
formation about the penalty approach. In section 4 , 5 and 6 we give respectively the principle of the proposed method, the based algorithm and the new solution. The simulation results of a three DOF robot arm are given in section 7 . Finally, some conclusions are drawn in section 8.

\section{Kinematic formulations}

Let $\theta$ be a $n \times 1$ vector of joint angles and $x$ a $m \times 1$ vector of the corresponding Cartesian coordinates of the end-effector position $(n<m), r=n-m$ being the degree of redundancy. Then $x$ and $\theta$ are related by the forward kinematic transformation $f(\cdot)$ which is a well-known non linear function:

$$
x=f(\theta)
$$

By differentiating Eq.1 with respect to time and defining $\dot{x}=\frac{\partial x}{\partial t}, \dot{\theta}=\frac{\partial \theta}{\partial t}$ and $J=\frac{\partial f}{\partial \theta}$, the following equation is obtained:

$$
\dot{x}=J \dot{\theta}
$$

For redundant manipulators where $m<n, J(\theta)$ is not a square matrix. Hence, there may exist an infinite number of joint velocities $\dot{\theta}$ satisfying Eq. 2 for each velocity vector $\dot{x}$

A unique joint velocity vector for each Cartesian velocity vector can be obtained by means of a MoorePenrose pseudoinverse. If $\operatorname{rank}(J)=m$, then a particular solution to Eq. 2 is given by:

$$
\dot{\theta}=J^{+}(\theta) \dot{x}
$$

By adding a null space vector to the right-hand side of the Eq.3, the general solution to Eq.2 becomes:

$$
\dot{\theta}=J^{+}(\theta) \dot{x}+\left(I-J^{+}(\theta) J(\theta)\right) v
$$

where $I$ is the identity matrix, $\left(I-J^{+}(\theta) J(\theta)\right)$ is the null space projection matrix, $v$ is an arbitrary joint velocity vector. $\left(I-J^{+}(\theta) J(\theta)\right) v$ is a homogeneous solution of $\mathrm{Eq} 2$ in the null space of $J$ that is orthogonal to $J^{+}$. This approach is however time consuming and non appropriate in the case of singularities. For non-redundant manipulator, the pseudoinverse is the common inverse $J^{-1}$. However, as the arm approaches singularities, $J(\theta)$ becomes ill conditioned and the numerical inverse solution leads to very large joint velocities.

Extended task-space formulation is an alternative means of solving the redundancy by including ( $n-$ $m$ ) additional constraints into the direct kinematic model. The following $(n-m)$ constraint vector is an example for optimizing a scalar kinematic objective function $\Phi(\theta)$.

Let us consider an extended objective function:

$$
\dot{\psi}(\theta, \lambda)=\Phi(\theta)+\lambda^{T}(x-f(\theta))
$$

$\lambda$ is the $(m \times 1)$ Lagrangian multiplier vector. The necessary conditions of optimality is given by:

$$
\frac{\partial \psi}{\partial \theta}=0 \Rightarrow \frac{\partial \Phi}{\partial \theta}=\left(\frac{\partial f}{\partial \theta}\right)^{T} \lambda=J^{T} \lambda
$$

and

$$
\frac{\partial \psi}{\partial \lambda}=0 \Rightarrow x=f(\theta)
$$

By multiplying Eq.6 by $N$ (the $n \times(n-m)$ null space matrix of $J, J N=0$ ), we obtain the necessary condition of optimality:

$$
N^{T} \frac{\partial \Phi}{\partial \theta}=0
$$

If the objective function $\Phi(q)$ is convex then the condition Eq. 8 becomes the necessary and sufficient condition of optimality.

One method to solve the inverse kinematic problem of redundant arms is to formulate it as an optimization problem with constaints, as follows:

$$
\left(\begin{array}{c}
\text { Minimize } \Phi(\theta) \\
\text { subject to } x_{d}(t)-f(\theta)=0 \text { and } h(\theta) \preceq 0
\end{array}\right.
$$

where $\Phi(\theta)$ is a scalar kinematic objective function of the joint angles to be minimized, $x_{d}(t)$ is the desired end-effector trajectory and $h(\theta)$ is the inequality constraint vector. Penalty functions convert the constrained optimization problem Eq.9 into an unconstrained problem.

\section{The penality-function method}

The algorithms usually proposed to solve constrained optimization problems can be divided into two categories: boundary-following methods and penalty-function methods. Within the first, we find, for example, the feasible-directions method and the gradient-projection method. These techniques switch directions from the gradient of the objective function to the gradient of the violated constraint whenever a move outside the feasible region occurs. The penalty-function methods lead to safer computational algorithms compared to gradient methods, with less computations, since they convert constrained minimization Eq.9 into unconstrained optimization of an augmented objective function. The constraints $h_{i}(\theta)$ can be equality or inequality constraints: 
In this case, the augmented objective function is:

$$
\Omega(\theta)=\Phi(\theta)+\sum_{i=1}^{l} \alpha_{i} p\left(h_{i}(\theta)\right)
$$

a) Exterior penalty function : $p\left(h_{i}\right)=h_{i}^{2} \Gamma\left(h_{i}\right)$ where $\Gamma\left(h_{i}\right)$ is the Heaviside function. The solution is outside the feasible region and tends towards the limit as the $\alpha_{i}$ penalty parameters increase. In this case we have:

$$
\begin{gathered}
\Omega(\theta)=\Phi(\theta)+\sum_{i=1}^{l} \alpha_{i} h_{i}^{2} \Gamma\left(h_{i}(\theta)\right) \\
\frac{\partial \Omega(\theta)}{\partial \theta}=\frac{\partial \Phi(\theta)}{\partial \theta}+\sum_{i=1}^{l} \alpha_{i} h_{i}^{2} \frac{\partial h_{i}(\theta)}{\partial \theta} \Gamma\left(h_{i}(\theta)\right)
\end{gathered}
$$

b) Interior penalty function : $p\left(h_{i}\right)=\frac{-1}{h_{i}}$. The solution is always inside the feasible region and tends towards the limit as the $\alpha_{i}$ decrease. In this case we obtain:

$$
\begin{gathered}
\Omega(\theta)=\Phi(\theta)+\sum_{i=1}^{l} \alpha_{i} \frac{-1}{h_{i}(\theta)} \\
\frac{\partial \Omega(\theta)}{\partial \theta}=\frac{\partial \Phi(\theta)}{\partial \theta}+\sum_{i=1}^{l} \alpha_{i} \frac{1}{\left(h_{i}(\theta)\right)^{2}} \frac{\partial h_{i}(\theta)}{\partial \theta}
\end{gathered}
$$

\section{Principle of the proposed method}

Our approach is based on neural network, it consists in training on-line a Widrow-Hoff neural network to solve the inverse kinematic problem. For this purpose, we introduce an extended position vector $X$ as:

$$
X=F(\theta)=\left[\begin{array}{l}
x \\
z
\end{array}\right]=\left[\begin{array}{l}
x \\
0
\end{array}\right]=\left[\begin{array}{l}
f(\theta) \\
g(\theta)
\end{array}\right]
$$

- $f(\theta) \in \Re^{m}$ is the forward kinematic vector defined in $(1)$.

- $g(\theta) \in \Re^{r}$ is the constraint vector added in the redundant case. These constraints can be represented by the general form:

$$
g(\theta)=N^{T} \frac{\partial \Omega(\theta)}{\partial \theta}=0
$$

where $N$ is the $n \times(n-m)$ null space matrix of $J$ which corresponds to the self motion of a redundant arm:

$$
N=\operatorname{det}\left(J_{a}\right)\left[\begin{array}{c}
J_{a}^{-1} J_{b} \\
-I_{n-m}
\end{array}\right]=\left[\begin{array}{c}
A J_{b} \\
-\operatorname{det}\left(J_{a}\right) I_{n-m}
\end{array}\right]_{(17)}
$$

where $J_{a}^{-1}=A / \operatorname{det}\left(J_{a}\right), J_{a}$ is a $m$-square matrix made of the $m$ first columns of $J$ and $J_{b}$ a $m \times(n-m)$ matrix of the remaining columns: $J=\left[\begin{array}{ll}J_{a} & J_{b}\end{array}\right]$, $A$ is the cofactor matrix of $J_{a}^{T}$, and $I_{n-m}$ is the $n-m$ identity matrix.

\subsection{Minimization of performance function subject to obstacles avoidance}

When the redundancy is utilized to avoid collision with a workspace object, the distance between the object and the closest robot link $\beta(\theta)$ should exceed a certain threshold $c$, which leads to an inequality constraint of the form

$$
h_{i}(\theta)=\dot{c_{i}}-\beta_{i}(\theta) \preceq 0, i=1, \cdot \cdot l
$$

where $\beta_{i}$ is a kinematic function of the joint angles $\theta$ and $c_{i}$ is a constant. For each inequality in Eq.18, two modes of operation are possible depending on $\theta$ and $c_{i}$ :

- Case One: $h_{i}(\theta) \preceq 0$

- In this case, the inequality constraint is satisfied and can be ignored. Therefore, the manipulator redundancy can be used to achieve the objective function $\Phi(\theta)$ while tracking the desired trajectory $x_{d}(t)$.

- Case One: $h_{i}(\theta) \succ 0$

- In this case, the inequality constraint is active, and the redundancy is utilized to satisfy the constraint and minimize an objective function $\Phi(\theta)$ while tracking the desired trajectory.

By applying Eq.(10) we obtain:

-Without inequality constraints:

In this case, $\Omega(\theta)=\Phi(\theta)$ is simply the scalar kinematic objective function. we have for each component $\mathrm{j}$ :

$$
\frac{\partial \Omega(\theta)}{\partial \theta_{j}}=\frac{\partial \Phi(\theta)}{\partial \theta_{j}}
$$

-With inequality constraints:

From (14) and (12), we have respectively for each component $j$

a) Exterior penalty method:

$$
\frac{\partial \Omega(\theta)}{\partial \theta_{j}}=\frac{\partial \Phi(\theta)}{\partial \theta_{j}}+2 \alpha_{j}\left(c_{j}-\beta_{j}(\theta)\right) \frac{-\partial \beta_{j}(\theta)}{\partial \theta_{j}} \Gamma\left(c_{j}-\beta_{j}(\theta)\right)
$$

b) Interior penalty method:

$$
\frac{\partial \Omega(\theta)}{\partial \theta_{j}}=\frac{\partial \Phi(\theta)}{\partial \theta_{j}}+\alpha_{j} \frac{1}{\left(c_{j}-\beta_{j}(\theta)\right)^{2}} \frac{-\partial \beta_{j}(\theta)}{\partial \theta_{j}}
$$

\section{The based algorithm}

For a given desired Cartesian position, the objective is to approximate the kinematic inverse model by a linear function at each trajectory point i.e. to 
find $\theta$ which sat isfies the forward mapping $x=f(\theta)$ while optimizing the given performance index $\Phi(\theta)$ and satisfying the additional constraints.

At each iteration $c$ for $k^{\text {th }}$ point, this algorithm involves two phases:

During the first phase, the input $X_{k}^{d}$ (the desired extended end-effector position) is presented and propagated through the network to compute the output value:

$$
\begin{gathered}
\theta_{k}^{c}=\theta_{d_{k-1}}+\tau W_{k}^{c} X_{k}^{d} \\
\left(\theta_{k}^{c}\right)_{i}=\left\{\begin{array}{l}
2 \pi+\gamma \text { if }\left(\theta_{k}^{c}\right)_{i} \succ 2 \pi \\
\left(\theta_{k}^{c}\right)_{i} \text { if }\left(\theta_{k}^{c}\right)_{i} \preceq 2 \pi \\
-2 \pi+\gamma \text { if }\left(\theta_{k}^{c}\right)_{i} \prec-2 \pi
\end{array}\right.
\end{gathered}
$$

where $\left(\theta_{k}^{c}\right)_{i}$ is the $i^{\text {th }}$ component of $\theta_{k}^{c}, \gamma$ is a random variable having a small variance to avoid the possible cyclic trajectory of the joint position, $\theta_{d_{k-1}}$ is the desired joint position obtained for the $(k-1)^{t h}$ point which is considered as the bias of the neural network and $\tau$ is the sampling period. The initial value $W_{k}^{0}$ is the optimal weight matrix $W_{k-1}^{*}$ obtained for the $(k-1)^{t h}$ point.

Then, the vecor $\theta_{k}^{c}$ is used to compute the endeffector position:

$$
X_{k}^{c}=F\left(\theta_{k}^{c}\right)
$$

and the error is given by:

$$
E_{k}^{c}=X_{k}^{d}-X_{k}^{c}=\left[\begin{array}{l}
\varepsilon_{k}^{c} \\
e_{k}^{c}
\end{array}\right]
$$

During the second phase, the weights of the neural network is adjusted according to the delta rule :

$$
W_{k}^{c+1}=W_{k}^{c}+\eta \triangle W_{k}^{c}
$$

where $\eta$ is the reduction factor $(0 \prec \eta \prec 2)$.

In the previous works, the search direction involved an inverse Jacobian matrix calculation. This solution is time consuming and problems may arise in the vicinity of singular points.

\section{A new solution}

We propose here a new method avoiding these drawbacks and leading to a very quick and efficient solution. The main idea is to use a Lyapunov function for calculating at each iteration the search direction $\triangle W$.

For a given initial weight matrix $W$, the algorithm updates $W$ iteratively in the direction of decreasing the Lyapunov function defined in terms of the errors:

$$
V=\frac{\lambda}{2} \varepsilon^{T} \varepsilon+\frac{1}{2} e^{T} e
$$

where $\varepsilon=x^{d}-x=x^{d}-f(\theta), e=0-g(\theta), x_{d}$ is the desired value of $x$ and $\lambda$ is the Lagrangian multiplier. The purpose of this multiplier is to force $\varepsilon$ to converge to zero by increasing $\lambda$ exponentially when $\theta$ is near the solution of $\varepsilon$.

For sake of simplicity, we eliminate in the following equations the index $k$ and $c$.

The time derivative of the Lyapunov function Eq.27 is given by:

$$
\begin{gathered}
\dot{V}=\left(\frac{\partial V}{\partial \theta}\right)^{T} \dot{\theta}+\left(\frac{\partial V}{\partial \lambda}\right) \dot{\lambda} \\
\dot{V}=-\left(\lambda J^{T} \varepsilon+J_{e}^{T} e\right)^{T} \dot{\theta}+\frac{1}{2}\|\varepsilon\|^{2} \dot{\lambda}
\end{gathered}
$$

where $J=\frac{\partial f(\theta)}{\partial \theta}$ and $J_{e}=\frac{\partial g(\theta)}{\partial \theta}$, by differentiating Eq.22, we obtain $\dot{\theta}=\tau \dot{W} X^{d}$. For simulation on a digital computer, we use a discrete time update rule: $\dot{W} \simeq \frac{\Delta W}{\tau}$ and $\dot{\lambda}=\frac{\Delta \lambda}{\tau}$. Then, Eq.29 becomes:

$$
\dot{V}=-\left(\lambda J^{T} \varepsilon+J_{\mathrm{e}}^{T} e\right)^{T} \Delta W X^{d}+\frac{1}{2 \tau}\|\varepsilon\|^{2} \Delta \lambda
$$

If we set:

$$
\dot{V}=-\frac{\lambda}{2}\|\varepsilon\|^{2}
$$

Then we can deduce the correction terms $\Delta W$ and $\triangle \lambda$ :

$$
\begin{aligned}
\Delta W & =X_{1}\left(\lambda J^{T} \varepsilon+J_{e}^{T} e\right) \frac{S G N\left(X^{d}\right)^{T}}{\left(X^{d}\right)^{T} S G N\left(X^{d}\right)}(32) \\
X_{1} & =\frac{\frac{\lambda}{2}\|\varepsilon\|^{2}+\frac{1}{2 \tau}\|\varepsilon\|\|e\|}{\left\|\lambda J^{T} \varepsilon+J_{e}^{T} e\right\|^{2}}
\end{aligned}
$$

and

$$
\Delta \lambda=\frac{\|e\|}{\|\varepsilon\|}
$$

where

$$
\begin{gathered}
\operatorname{SGN}(X)=\left[\begin{array}{c}
\operatorname{sgn}\left(x_{i}\right) \\
\vdots \\
\operatorname{sgn}\left(x_{n}\right)
\end{array}\right] \\
\operatorname{sgn}\left(x_{i}\right)=\left\{\begin{array}{c}
+1 \text { if } x_{i}>0 \\
-1 \text { if } x<0
\end{array}\right.
\end{gathered}
$$

Substituting Eq.32 and Eq.33 in Eq.30 we obtain Eq.31. This result implies that $\dot{V} \prec 0 \forall \varepsilon \neq 0$ and $\dot{V}=0$ iff $\varepsilon=0$.

The update of $W$ based on $\triangle W$ determined by Eq. 32 guarantes the convergence. The weights of the neural network and the Lagrangian multiplier are adjusted according to

$$
W_{k}^{c+1}=W_{k}^{c}+\eta_{1} \Delta W_{k}^{c}
$$




$$
\lambda^{c+1}=\lambda^{c}+\eta_{2} \triangle \lambda
$$

where $\eta_{i}$ is the reduction factor $(0 \prec \eta \prec 2)$.

We must emphasize that no Jacobian inverse matrix calculation are necessary here. Furthermore, if the desired trajectory does not present large discontinuities, each new desired point $X_{k}^{d}$ is very close of the previous $X_{k-1}^{d}$ and the error is small. This algorithm converges very quickly towards the solution $\theta_{d_{k}}$ with few operations per iteration.

\section{Simulation}

A 3DOF planar robot is considered to show the validity of our method. The desired path used in our simulation is given by:

$$
x_{d}=\left(\begin{array}{c}
x_{1 d} \\
x_{2 d}
\end{array}\right)=\left(\begin{array}{c}
3 \cos (2 \pi t) \\
\sin (2 \pi t)
\end{array}\right)
$$

The forward kinematic function is:

$$
\begin{aligned}
f(\theta) & =\left(\begin{array}{l}
x_{1} \\
x_{2}
\end{array}\right) \\
& =\left(\begin{array}{l}
l_{1} c_{1}+l_{2} c_{12}+l_{3} c_{123} \\
l_{1} s_{1}+l_{2} s_{12}+l_{3} s_{123}
\end{array}\right)
\end{aligned}
$$

and the $(n-m)$ null space vector of $J$ is :

$$
N=\left[\begin{array}{c}
-l_{2} l_{3} s_{3} \\
l_{1} l_{3} s_{23}+l_{2} l_{3} s_{3} \\
-l_{1} l_{2} s_{2}-l_{1} l_{3} s_{23}
\end{array}\right]
$$

where $s_{i j}=\sin \left(\theta_{i}+\theta_{j}\right), c_{i j}=\cos \left(\theta_{i}+\theta_{j}\right)$ and the link lengths are $l_{1}=1.5 m, l_{2}=1 m, l_{3}=0.5 \mathrm{~m}$. Any convex objective function may be used in our approach. Here, we have taken $\Phi(\theta)=\frac{1}{2} \sum_{i=1}^{3} \theta_{i}^{2}$.

\section{The first simulation.}

- In the first simulation, we consider the endeffector motion on the environment surface (figure1 $(a)$ ) represented here by the straight line:

$y-a x-b=0$ with $a=-1$ and $b=2,(x, y)$ are the Cartesian coordinates in the base frame.

We decide to minimize the objevtive function $\Phi(\theta)$ defined above and ensure that the arm will not go over the contact surface. To this end, we maintain a constant distance $\beta_{0}$ between the last link position $\left(x_{2}, y_{2}\right)=\left(l_{1} c_{1}+l_{2} c_{12}, l_{1} s_{1}+\right.$ $\left.l_{2} s_{12}\right)$ and the contact surface. The additional constraint is $h(\theta)=\beta(\theta)-\beta_{0} \preceq 0$ with $\beta(\theta)=$ $y_{2}-a x_{2}-b$.
- Figurel $(b)$ exhibit the arm configuration for the objective function $\Phi(\theta)$ without inequality constraints. The end-effector coordinates track very closely the desired trajectory. However these configuration are not attainable in practice.

- By applying inequality constraints and using the interior penalty method ( $\alpha=0.01$ ), the arm configuration during the task is seen in figure1 $(c)$. While the end-effector moves, the objective function is well minimized and the last link position remains always inside the feasible region and far from the frontier of the allowable workspace.
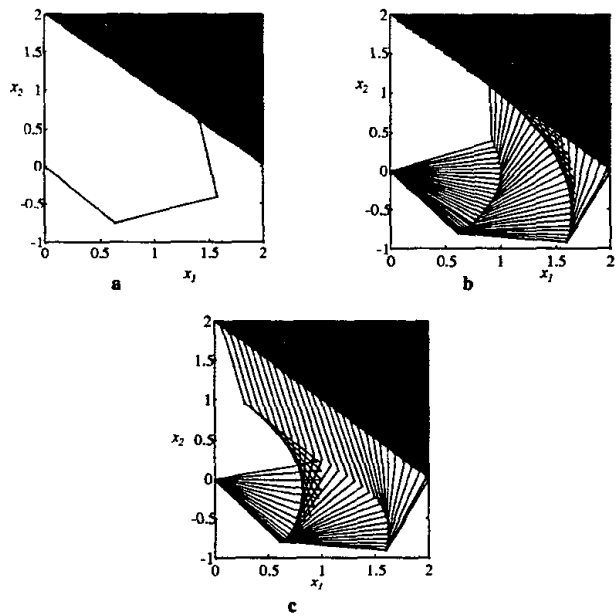

Figure 1: (a) End-effector motion on the environment surface, (b) Arm configurations using $\Phi(q)$ without constraints, (c) Arm configurations using $\Phi(q)$ with constraints

\section{The second simulation.}

- In the second simulation, we fixe the end-effector at a given position $(x, y)=(0.7 m, 1.8 m)$, then we move the obstacle object which is a circle with radius $c=0.1 m$ towards the robot, we obtain figure2(a). The same inequality constraint as in the previous case is used (i.e. the distance between the center of obstacle and the manipulator segments will be greater or equal to zero). The manipulator change its configurations in real time to avoid the moved obstacle while keeping his end-effector at a fixed position figures $2(b, c$ and $d)$. 

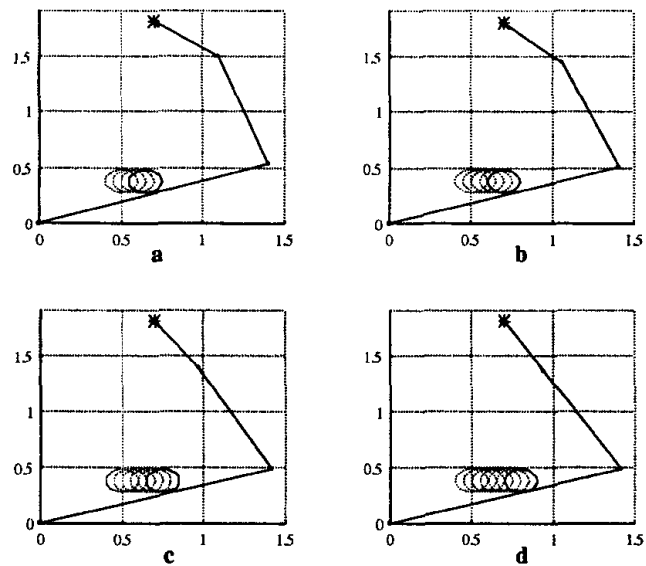

Figure 2: - (a) The end-effector is fixed at Cartesien position and the obstacle is moved; In (b), (c) and (d) Manipulator change its joint configurations to stay at the fixed Cartesian position while avoiding the obstacle.

\section{Conclusion}

We have presented an efficient local approach for the trajectory tracking and joint path generation of redundant robot manipulator. Moreover, it can be applied to non-redundant manipulators. Our method is based on formulating a simple optimization problem using neural network. This problem is considered as a minimization of a Lyapunov function defined in terms of position error and any other criterion evolution error. The neural network is adapted in the direction of decreasing the Lyapunov function to move the end effector to the desired position while minimizing an objective function and avoiding a collision with a workspace object or a contact surface environment. This method achieves an accurate solution with only a few iterations per input point even in singular points and requires only the computation of the direct kinematic functions.

\section{References}

[1] K. H. Hunt, "Robot kinematics- a compact analytic inverse solution for velocities," ASME Journal of Mechanisms, Transmissions and Automation in Design, vol. 109. Nø., p. $42,1987$.

[2] M. Kircanski and T. Petrovic, "Combined analyticalpseudoinverse kinematic solution for simple redundant manipulators and singularity avoidance," International Journal of Robotics Research, vol. 7. Nø.1., p. $188,1993$.

[3] Y. Bestaoui, "An unconstrained optimization approach to the resolution of the inverse kinematic prob- lem of redundant and non-redundant robots," Robotics and Autonomous Systems, vol. 7. Nø., p. 37, 1991.

[4] R. Featherstone, "Accurate trajectory transformations for redundant and nonredundant robots," in Intermational Conference on Robotics and Automation, (San Diego, California), pp. 8-13, May 1994.

[5] M. Ait and A. C. Chevallereau, "Resolution of robot redundancy in the cartesian space by criteria optimization," in IEEE Conference on Robotics and Automation, (Atlanta), pp. 646-651, 1993.

[6] R. Mayorga, A. K. C. Wong, and K. S. Ma, "An effcient local approach for the path generation of robot manipulators," Journal of Robotics Systems, vol. 7(1), no. 1, pp. 23-55, 1990.

[7] P. Chiacchio, S. Chiaverini, L. Sciavicco, and B. Siciliano, "Closed-loop inverse kinematics schemes for constrained redundant manipulators with task space augmentation and task priority strategy," The International Journal of Robotics Research, vol. 10. Nø. 4., p. $410,1991$.

[8] H. Seraji and R. Colbaugh, "Improved configuration control for redundant robots," Joumal of Robotic Systems, vol. 7, no. 6, pp. 897-928, 1990. 